\title{
新冠肺炎防控期间PET/CT检查护理工作流程的优化与 防控措施
}

\author{
黄晓辉 ${ }^{1}$ 王荣福 ${ }^{1,2 *}$ 崔 媛 $^{1}$ 蔡春苗 $^{1}$ 李晓艳 ${ }^{1}$ 安 敏 $^{1}$ 李小东 $^{1}$ \\ 1. 北京大学国际医院核医学科, 北京 102206 \\ 2. 北京大学第一医院核医学科, 北京 100034
}

\begin{abstract}
摘 要: 核医学科PET/CT检查流程多、整过检查涉及多个环节, 对患者的管理要求比较复杂, 患者在注射放射 性药物后需要在相对封闭的环境中停留时间长，所以医护人员与患者之间、患者与患者之间交叉感染的风险大。在新 型冠状病毒肺炎 (NCP) 防控期间, 对进行PET/CT检查的患者, 制定防控应急预案, 对工作流程进行优化, 采取必要 的防控措施, 做好医务人员的个人防护, 对患者进行正确的健康宣教, 降低暴露风险, 减少交叉感染, 保证核医学检 查质量和安全是非常重要的。
\end{abstract}

关键词：新型冠状病毒防控; PET/CT；防控措施；患者宣教

\section{Optimization, Prevention and Control Measures of PET/CT Examination and Nursing Workflow During the Prevention and Control of Novel Coronavirus Pneumonia}

\author{
Xiao-Hui Huang ${ }^{1}$, Rong-Fu Wang ${ }^{1,2 *}$ Yuan Cui ${ }^{1}$, Chun-Miao Cai ${ }^{1}, X_{i a o-Y a n ~} \mathrm{Li}^{1}, \mathrm{Min} \mathrm{An}^{1}$, Xiao-Dong $\mathrm{Li}^{1}$ \\ 1. Department of Peking University International Hospital, Beijing 102206, China \\ 2. Department of Peking University First Hospital, Beijing 100034, China
}

\begin{abstract}
The PET/CT examination in nuclear medicine subject has many procedures involved many links and workplaces, and the management requirements for patients are complex. Patients need to stay in a relatively closed environment for a long time after the injection of radio pharmaceuticals, so the risk of cross-infection between medical staff and patients, and between patients is high. In novel coronavirus pneumonia (NCP) during the prevention and control, it's very important for patients carried out PET/CT examination to formulate emergency preplans for prevention, control and optimize the workflow, take necessary control measures, make the medical staff of personal protection. We have to do well for the patients of the correct health education, reduce exposure risk, reduce the cross infection, and guarantee the quality of nuclear medicine inspection and safety.
\end{abstract}

Keywords: Novel coronavirus prevention and control; PET/CT; prevention and control measures; patient education

\section{一、背景}

2019年12月以来，湖北省武汉市发现多起病毒性肺炎病例，均诊断为病毒性肺炎/肺部感染。2020年1月12日世界 卫生组织正式将造成武汉肺炎疫情的新型冠状病毒命名为 “2019新型冠状病毒 (2019-nCov) ”。国际病毒分类委员 会的冠状病毒研究小组 (CSG) 正式命名为 “SARS-CoV-2”。国家卫生健康委员会发布2020第1号公告, 将新型冠状

“通讯作者: 王荣福, 1955 年9月, 男, 汉族, 福建南平人, 现任北京大学第一医院教授, 主任医师, 博士生导 师; 北京大学国际医院核医学科主任, 医学和药学博士。研究方向: 临床与分子核医学、放射性药学。

基金项目: 北京科委首都临床特色应用研究基金项目（Z181100001718088）; 北京大学国际医院科研基金重点项 目（YN2019ZD03）；北京市科技计划项目（首都健康保障培育研究专项课题,Z181100001618017）；北京大学医-信 交叉项目（BMU2018MI009，BMU2018MI010); 国家重大科学仪器设备开发专项（2011YQ03011409）; 十二五国家 支撑项目基金（2014BAA03B03）。 
病毒 (2019-nCoV) 感染的肺炎 (Novelcoronavirus pneumonia, 简称: NCP) 纳人 《传染病防治法》规定的乙类传染 病，并采取甲类传染病的预防、控制措施 ${ }^{[1]}$ 。

\section{二、疫情防控期间核医学 PET/CT 检查特点}

PET/CT检查是肿瘤篮查, 诊断与分期, 疗效评估和预后评价的重要方法, 其临床应用价值已得到肯定 ${ }^{[2]}$ 。为了保证 临床工作需求, PET/CT日常检查在新型冠状病毒感染肺炎流行期间没有停止。尤其是现在境外输人新冠肺炎新发病例应 引起高度重视。众所周知, PET/CT检查的特点是检查流程涉及的环节多、过程复杂、患者在相对封闭的环境中停留时间 长、与工作人员接触的频次多等 ${ }^{[3]}$ 。如果是新型冠状病毒携带者，检查过程中导致工作人员感染的概率比较高。

在疫情防控期间, 根据各大医院规定标准, 所有进人医疗机构的人员必须做到 $100 \%$ 填写新冠肺炎流行病学调查 表和监测体温, 体温 $\geqslant 37.2^{\circ} \mathrm{C}$ 且非专科疾病可以解释的发热患者, 应先到发热门诊就诊篎查, 排除相关传染病后回专 科就诊 ${ }^{[4]}$, 经过预检分诊, 到核医学科就诊者均已经过临床科室篮查。因此, 在PET/CT检查的全流程中防御的重点是 要识别出具有病毒感染风险者, 在检查过程中对其实施严格管理, 将工作人员和其他受检者发生感染的风险或概率降 到最低。

\section{三、疫情防控期间 PET/CT 检查护理工作流程的优化与防控措施}

在医院整体防御的框架下, 医院感染科专家到核医学科现场评估后, 给出了新冠肺炎防控期间核医学检查过程中 防护的指导性意见。反复模拟检查流程中各个环节所涉及的问题, 经专业人员共同分析和讨论后, 根据国家卫生健康 委员会发布2020第1号公告及北京市核医学质量控制和改进中心, 新型冠状病毒肺炎疫情防控期间核医学影像检查防 控方案 (2版), 制定了北京大学国际医院新型冠状病毒疫情防控期间核医学感染防控应急处理预案（PKUIH-HYXIII-SOP-18)。

\section{(一) 疫情防控期间受检者预约就诊防控措施}

1. 在新型冠状病毒肺炎疫情防控工作开展期间

门急诊严格实行三级预检分诊制度, 医院人口设置预检分诊处对所有进人医院的人员进行新型冠状病毒肺炎的预 检分诊。患者在门诊完成第1次篮查表填写及体温测量, 到达核医学科由预约分诊护士完成第2次篮查表及体温测量, 到达诊室或检查室, 接诊医生再次询问患者有无发热, 并对《门急诊人员篮查登记表》内容再次复核、签字、留存, 并在门急诊病历中进行流行病学相关记录。第三方工作人员、配送、陪检人员进科室也需要填写笁查表, 预约分诊护 土每日收集并汇总《门急诊人员篮查登记表》, 将正常、异常分开装订、统计数量并做好相应记录。

2. 预约时详细询问受检者及家属流行病史, 对受检者分类安排预约检查

为将病毒感染的风险降到最低, 在疫情防控关键阶段, 根据临床需求和患者病情, 预约分诊护士通过电话确认并 采取有效沟通, 将需要进行核医学影像检查的患者进行分类, 具体包括:

（1）对于择期检查的外地患者、返京后隔离不满14天的患者，推迟检查。

（2）新冠肺炎确诊患者、疑似患者、伴有原因不明的发热、咳嗽、腹泻者，推迟检查。

(3) 无特殊情况者按照正常流程安排检查。

（4）控制每日检查数量，避免人员聚集，减少交叉感染。

（二）疫情防控期间预约就诊患者宣教

1. 疫情防控期间, 进院人口时间会因防控篎查而导致延长, PET/CT 显像剂具有快速衰变的自然属性, 请受检者 按预约时间提前到医院人口进行篎查，避免延误正常检查。

2. 备孕、孕妇及14岁以下未成年人禁止陪同患者做此项检查。育龄期女性否认其备孕、怀孕及哺乳期。如临床必 需，哺乳期妇女检查结束后应停止哺乳至少数小时，在此期间远离婴幼儿。

3. 疫情防控期间不建议家属陪检, 特殊患者, 如儿童、老年人、行动不便者、残疾等患者, 为防范患者跌倒、坠 床等，检查当日允许有一名得到授权的家属负责换扶、陪伴和看护等，并要采取适当的辐射防护措施。

(三) 疫情防控期间PET/CT检查前护理防控措施

1. 检查当日预约分诊护士再次对受检者及其家属进行流行病史篮查, 包括测量患者体温, 了解近期 (14天) 居住 地、旅行史、接触史等, 遇有可能感染新冠病毒的情况, 立即启动北京大学国际医院门诊发现疑似病例应急预案。

2. 受检者对所提供的流行病史的真实性负法律责任，并签署承诺书。 
3. 工作人员需佩戴外科防护口罩 (必要时佩戴N95口罩) 、医用帽、护目镜和手套, 穿隔离衣。有辐射防护要求 时加穿铅衣、铅围脖、铅眼镜 ${ }^{[3]}$ 。

4. 候诊受检者大间距排队等候, 保持 1 米的距离, 安排患者及其陪同人员分区, 疏离候诊, 与接诊医师保持安全 距离。

5. 为避免交叉感染，按预约顺序分批次引导受检者进注射室。

（四）疫情防控期间PET/CT检查前患者宣教

1. 为切断病毒传播途径，指导受检者及陪护家属在候诊和检查过程中均需佩戴一次性医用外科口罩 ${ }^{[6]}$ 。

2. 该检查使用放射性药物，患者经医师接诊后，在预约分诊护士引导下到候诊区等待注射药物，不要随意走动， 避免在公共区域停留，不与他人聚集，保持距离。

3. 受检者家属如无特殊情况尽量不要进人候诊室或显像室，患者需要照顾除外。

(五) 疫情防控期间PET/CT检查期间护理防控措施

1. 注射护士佩戴外科防护口罩 (必要时佩戴N95口罩)、医用帽、鞋套、穿隔离衣、个人剂量笔、穿铅衣、戴铅 帽、铅围脖、戴双层乳胶手套。

2. 严格执行 “三查、八对” 核对制度, 受检者分批次注射, 确认各项信息无误, 核对检查项目及注射的显像剂剂 量，避免差错，随时进行手卫生。

3. 严格执行无菌操作, 做到 1 人 1 巾 1 带, 在安全距离下进行操作, 测血糖时与受检者隔室在操作台内操作, 测量 结束及时对血糖仪用 $75 \%$ 酒精进行表面消毒。

4. 放射性药物注射完毕, 做好注射后宣教, 清理用物, 做好垃圾分类: 将使用后的放射性物品（包括手套、套袖 等) 统一放人到放射性防护垃圾桶内衰变, 医疗垃圾、生活垃圾分类放置, 用有效氯为 $1000 \mathrm{mg} / \mathrm{L}$ 的含氯消毒进行擦 拭消毒操作台面 ${ }^{[7]}$ 。

5. 视频监控注射后休息室, 受检者注射后候检分区, 大房间不超过 3 人, VIP房间单独 1 人, 房间内配置话筒及呼 叫器。

6. 检查期间给予患者戴一次性手套, 穿一次性鞋套, 检查床铺用一次性床单。检查完毕, 将上述防护用品放人医 疗废物桶 ${ }^{[8]}$ 。

（六）疫情防控期间PET/CT检查期间患者宣教

1. 指导受检者在注射后休息室指定区域等候检查, 安静休息, 不与他人交谈、聚集, 如有不适及时按呼叫器。

2. 指导受检者按检查需要饮水，饮水时可以摘口罩，饮水后立即戴口罩。

3. 如有打喷德、咳嗽请使用纸巾按住口鼻, 不随地吐痰, 使用后的东西放人医疗垃圾桶。

4. 等待检查期间不需要憋尿, 使用卫生间马桶后多冲水, 切勿污染马桶外面及自己的衣物, 避免交叉感染。

（七）疫情防控期间PET/CT检查结束后护理防控措施

1. 接触患者后, 严格执行手卫生消毒, 杜绝戴接触患者的手套操作键盘、鼠标及接触其他物品。

2. 注射室每日采用紫外线消毒，时间不少于 $30 \mathrm{~min}$ ，紫外线灯管用 $75 \%$ 酒精擦拭消毒。

3. 受检者检查完成后按指定路线离开，严格实施并特别强化受检患者与工作人员双通道管理。

4. 注射后休息室的床单位及被服及时更换。每日常规进行空气消毒、地面消毒和物件表面消毒, 可根据患者的数 量适当增加消毒频次。

5. 注射护士离开注射室进人其他区域时，需要脱掉鞋套、隔离衣，并及时进行淋浴，更换清洁工作服。

( 八 ) 疫情防控期间PET/CT检查结束后患者宣教

1. 检查结束 24 小时以内避免接触备孕、孕妇以及婴幼儿, 要跟上述人群保持 2 米以上的距离, 减少与家人的近距 离密切接触, 做好手卫生, 避免外出活动。

2. 检查结束后医师确认可以离开，方可沿指定路线离开，不可返回就诊区域。

3. 门诊取报告时按照预约就诊流程完成相关体温测量及填写流行病史篮查表。

四、门诊发现符合病例定义的疑似病例时报告工作流程及防控措施

(一) 报告管理 
新型冠状病毒肺炎纳人 《中华人民共和国传染病防治法》规定的乙类传染病, 并采取甲类传染病的预防、控制措 施，按要求须于诊断疑似病例（北京大学国际医院专家组会诊）后2小时内上报。

(二 ) 疫情防控期间核医学科PET/CT预约检查防控应急措施流程图（见图1）

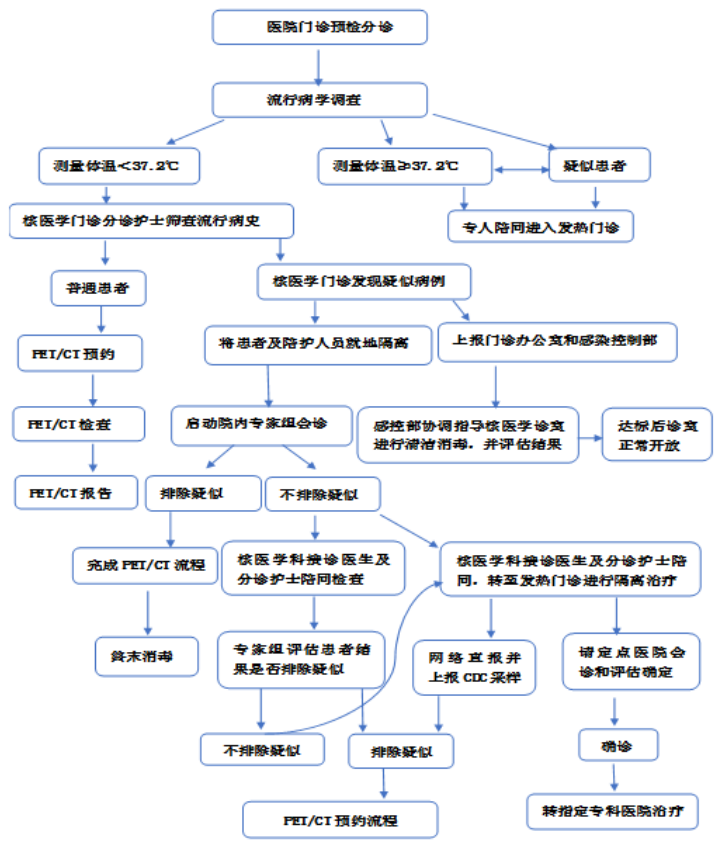

图1 新型冠状病毒疫情期间核医学PET/CT预约检查防控应急措施流程图

（三）疑似患者收治区域的消毒防控措施

1. 地面、墙壁、门窗

有效氯含量为 $1000 \mathrm{mg} / \mathrm{L}$ 的含氯消毒剂或 $0.2 \%$ 过氧乙酸溶液喷酒或擦拭消毒 ${ }^{[9]}$ 。

2. 家具、物体表面

可用 $0.2 \%$ 过氧乙酸溶液或有效氯为 $1000 \mathrm{mg} / \mathrm{L}$ 的含氯消毒进行浸泡、喷酒或擦拭消毒。

3. 核医学设备

在检查过程中患者可能接触的部分（探头、机架）同样消毒，使用 $75 \%$ 的乙醇擦拭。如有污物或肉眼可见污渍， 先使用一次性吸水材料完全清除污渍后，再行消毒。

4. 其他耐湿、耐腐蚀物品

$1000 \mathrm{mg} / \mathrm{L}$ 含氯消毒剂浸泡或擦拭消毒。

5. 不耐腐蚀物品

可用 $75 \%$ 酒精擦拭或浸泡消毒。

6. 疑似患者停留过的房间

采用紫外线消毒，方法：紫外线照射消毒有效距离为 $1.5 \mathrm{~W} / \mathrm{M}$ ，需合理配置覆盖整个房间，时间不少于 $30 \mathrm{~min}$

五、疫情防控期间失分化型甲状腺癌患者 PET/CT 显像后拟行 ${ }^{131} 1$ 治疗前的护理防控措施与健康宣教

甲状腺癌是常见的内分泌系统肿瘤, 以分化型甲状腺癌 ( DTC) 最为多见。通过甲状腺全切或次全切术, ${ }^{131}$ I和口 服TSH抑制剂量的甲状腺素联合治疗，多数DTC患者可以获得较好的治疗效果，但仍有 $2 \% \sim 5 \%$ 的患者在治疗过程或 疾病的自然进展中会出现肿瘤细胞失分化现象 ${ }^{[10]}$ 。失分化型甲状腺癌患者在行PET/CT检查后, 经常会需要继续行 ${ }^{131} \mathrm{I}$ 治疗，根据我国相关法规， ${ }^{131}$ I治疗必须在符合辐射安全的核素病房内进行 ${ }^{[11]}$ ，在疫情防控期间， ${ }^{131}$ I治疗患者人院前 的防控措施及患者知识宣教尤其重要。

(一) 疫情防控期间拟行 ${ }^{131} \mathrm{I}$ 治疗人院前的防控措施

1. 严格执行预检分诊制度，核医学门诊预约分诊护士对拟行131I治疗的患者详细询问做完PET/CT检查后有无不 
适反应，近期2周内身体健康状况，有无咳嗽、发烧等情况。

2. 接诊医生根据患者情况开具住院证, 发现疑似或确诊NCP患者暂停办理人院, 并立即停用甲状腺激素, 待排除 肺炎或肺炎痊愈后再择期治疗。

3. 符合人院条件者开具住院证，安排至单人单间放射性核素病房。

(二) 疫情防控期间拟行 ${ }^{131}$ 治疗人院前患者宣教

1. 建议患者自行前往医院办理人院手续, 如需陪同，原则上不超过 1 人。

2. 指导患者做好个人防护，准备充足的生活用品及防护用品，在院期间必须全程佩戴口罩，做到勤洗手。

3. 核素病房实行 24 小时封闭管理，在院期间不允许家属探视，可随时使用视频、电话等与医护人员或家属联系， 减少交叉感染。

4. 指导患者在疫情防控期间要保持心情舒畅，不要有思想压力和精神包袱，积极做好人院前的各项准备工作。

\section{六、结束语}

在疫情防控关键阶段, 鉴于新冠病毒具有很强的传染性, 有的新冠肺炎患者潜伏期较长, 临床表现轻微, 为将病 毒感染的风险降到最低, 核医学PET/CT检查严格掌握适应证、临床需求和患者病情, 并通过采取有效沟通, 将非急 需的检查推迟进行, 力争做好防控前移。同时, 工作中落实预防和控制新冠病毒感染的总原则, 通过执行上述一系列 的优化工作流程和规范防控措施，在保证质量完成检查的同时，实现了受检患者和工作人员“零感染”。

\section{参考文献:}

[1]北京市核医学质量控制和改进中心.北京医学会核医学分会.《新型冠状病毒肺疫情防控期间间核医学影像检查 防控方案第二版》.2020,1-2.

[2]王荣福.PET/CT 肿瘤诊断学.北京: 北京大学医学出版社. 2008,171.

[3]王向青,余浩军, 顾宇参.复旦大学附属中山医院新冠肺炎防控期间核医学科PET检查防护方案(2020 v.1).中国临床 医学. 2020,27(1),16-19.

[4]北京大学国际医院.新型冠状病毒感染防控和医疗管理工作预案(第3版). 2020,12-13.

[5]中华预防医学会医院感染控制分会, 中国医院协会医院感染管理专业委员会和中国感染控制杂志编辑委员 会.《新冠肺炎疫情防控期间医疗机构不同区域工作岗位个人防护专家共识》.中国感染控制杂志. 2020.3(19)3:1-14.

[6]中国国家卫生健康委办公厅。《国家卫生健康委办公厅关于印发医疗机构内新型冠状病毒感染预防与控制技术 指南(第一版)的通知》.国卫办医函[2020]65号. 2020,1.22.

[7]中华人民共和国卫生部、《医疗机构消毒技术规范》(WS/T 367-2012). 2012,31-32.

[8]中国国家卫生健康委办公厅。《国家卫生健康委办公厅关于做好新型冠状病毒感染的肺炎疫情防控期间医疗机 构医疗废物管理工作的通知》.(国卫办医函国卫办医函[2020]81号). 2020,1.28.

[9]中华医学会核医学分会, 《中华核医学与分子影像杂志》编辑委员会. 2019新型冠状病毒感染疫情期间核医学诊 疗安全防控专家共识(第一版).中华核医学与分子影像杂志. 2020,40(3),133-134.

[10] 张英杰,林岩松, 梁军.甲状腺癌失分化机制的研究进展.中华核医学与分子影像杂志. 2014,34(4),327-328.

[11]王荣福,安锐主编.核医学.第九版,北京: 人民卫生出版社, 2018,240-241. 